\title{
Man-in-the-middle-attack: Understanding in simple words
}

\author{
Avijit Mallik $^{a^{*}}$, Abid Ahsan ${ }^{b}$, Mhia Md. Zaglul Shahadat ${ }^{a}$ and Jia-Chi Tsou ${ }^{c}$
}

${ }^{a}$ Department of Mechanical Engineering, RUET, Rajshahi-6204, Bangladesh

${ }^{b}$ Department of Computer Science and Engineering, RUET, Rajshahi-6204, Bangladesh

${ }^{c}$ Department of Business Administration, China University of Technology, Taipei City, Taiwan

CH R O N I C L E ABS T R A C T

Article history:

Received: September 18, 2018

Received in revised format: Octo-

ber 20,2018

Accepted: January 2, 2019

Available online:

January 2, 2019

Keywords:

MITM attack

Cyberattack

Crime

Media

\begin{abstract}
These days cyberattack is a serious criminal offense and it is a hotly debated issue moreover. A man-in-the-middle-attack is a kind of cyberattack where an unapproved outsider enters into an online correspondence between two users, remains escaped the two parties. The malware that is in the middle-attack often monitors and changes individual/classified information that was just realized by the two users. A man-in-the-middle-attack as a protocol is subjected to an outsider inside the system, which can access, read and change secret information without keeping any tress of manipulation. This issue is intense, and most of the cryptographic systems without having a decent authentication security are threatened to be hacked by the malware named 'men-in-themiddle-attack' (MITM/MIM). This paper essentially includes the view of understanding the term of 'men-in-the-middle-attack'; the current work is mainly emphasized to accumulate related data/information in a single article so that it can be a reference to conduct research further on this topic at college/undergraduate level. This paper likewise audits most cited research and survey articles on 'man-in-the-middle-attack' recorded on 'Google Scholar'. The motivation behind this paper is to help the readers for understanding and familiarizing the topic 'man-in-the-middle attack'.
\end{abstract}

\section{Introduction}

In cryptography and PC security, a man-in-the-middle attack (MITM) is an attack where the attacker furtively transfers and perhaps changes the correspondence between two parties who trust they are straightforwardly communicating with each other. A man in the middle (MITM) attack is a general term for when a culprit positions himself in a discussion between a client and an application; either to listen stealthily or to imitate one of the parties, making it show up as though an ordinary trade of information is in progress (Meyer \& Wetzel, 2004; Kish, 2006; Hypponen \& Haataja, 2007; Ouafi et al. 2008). The objective of an attack is to take individual information, for example, login certifications, account points of interest and charge card numbers. Targets are normally the clients of financial applications, SaaS businesses, web-based business locales and other sites where logging in is required. Information obtained during an attack could be utilized for many, purposes, including fraud, unapproved support exchanges or 
an unlawful watchword change. Furthermore, it can be utilized to gain a decent footing inside an anchored edge during the infiltration phase of an Advanced Persistent Threat (APT) strike. Fig. 1 portrays a schematic of 'men-in-the-middle-attack' belief system. A man-in-the-middle attack allows a malicious actor to intercept, send and receive data meant for someone else, or not meant to be sent at all, without either outside party knowing until it is too late. Man-in-the-middle attacks can be abbreviated in many ways, including MITM, MitM, MiM or MIM (Ouafi et al., 2008; Joshi et al., 2009; Khader \& Lai, 2016 ; Tung et al., 2016; Wallace \& Miller, 2017; Conti et al., 2016).

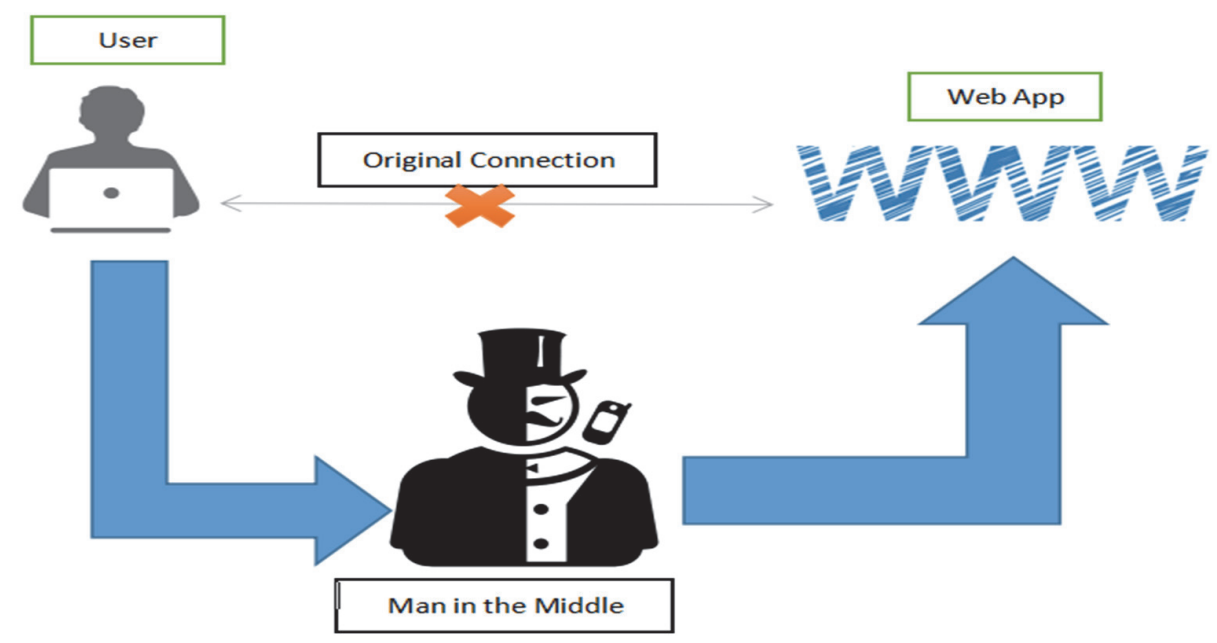

Fig. 1. Men-in-the-middle attack ideology schematic

One case of man-in-the-middle attacks is dynamic eavesdropping, in which the attacker makes independent associations with the victims and transfers messages between them to influence them to trust they are talking straightforwardly to each other over a private association when in certainty the whole discussion is controlled by the attacker. The attacker must have the capacity to intercept every single significant message passing between the two casualties and inject new ones. This is direct in many conditions; for instance, an attacker within gathering scope of an unencrypted wireless access point (Wi-Fi) could insert himself as a man-in-the-middle (Callegati et al., 2009; Desmedt, 2011). As an attack that goes for circumventing common authentication, or scarcity in that department, a man-in-the-middle attack can succeed just when the attacker can mimic every endpoint agreeable to them not surprisingly from the genuine closures. Comprehensively speaking, a MITM attack is what might as well be called a mailman opening your bank proclamation, writing down your record points of interest and after that resealing the envelope and delivering it to your entryway. Most cryptographic conventions include some type of endpoint authentication particularly to persist MITM attacks. For instance, TLS can authenticate one or the two parties using a commonly confided in endorsement expert (Sounthiraraj et al., 2014; Khader \& Lai, 2015; Rahim, 2017).

\section{Literature review}

MITM is named for a ball game where two people play catch while a third person in the middle attempts to intercept the ball. MITM is also known as a fire brigade attack, a term derived from the emergency process of passing water buckets to put out a fire. In the year 2004, U. Meyer and S. Wetzel presented a report on Universal Mobile Telecommunication System's (UITM) security protocol where they discussed about 'men-in-the-middle-attack' on mobile communication (Meyer \& Wetzel, 2004). In 2006, Kish published his research in a master listed journal where he showed an encryption method of MITM using Kirchhoff-loop-Johnson (-like)-noise cipher (Kish, 2006). Hypponen and Haataja (2007), made a 
research on secure Bluetooth communication and showed their developed system was capable of preventing MITM attack (Hypponen \& Haataja, 2007). Sun et al., 2018 and Saif et al., 2018; made similar type of researches on updated version of Bluetooth networks security and discussed about new techniques to prevent MITM in two party's communication (Sun et al., 2018; Saif et al., 2018). Ouafi et al. (2008), Callegati et al. (2009), Joshi et al., (2009), Desmedt, (2011) and Sounthiraraj et al., (2014) conducted researches about HTTP security and those researches found MITM as a very serious threat and those also discussed about the prevention techniques (Ouafi et al., 2008; Callegati et al., 2009; Joshi et al., 2009; Desmedt, 2011; Sounthiraraj et al., 2014). Khader et al. (2015) and Tung et al. (2016) published their researches which mostly talks about different prevention methods of MITM (Khader \& Lai, 2015; Tung et al., 2016). Wallace and Miller (2017) patented their research about endpoint based MITM where they tested multiple prevention methods for MITM (Wallace \& Miller, 2017). Conti et al. (2016); did a survey on MITM and its effects on the economy. Li et al. (2017), Rahim (2017) and Howell et al. (2018) made identical researches on prevention of MITM mainly for internet communication and those papers discusses several unique and effective measures on prevention of MITM from on-net communication (Li et al., 2017; Rahim, 2017; Howell et al., 2018). Fei et al. (2017), Usman et al. (2018), Valluri (2018) and Kuo et al. (2018) published their review reports on MITM which mostly discusses about WLAN security for 2-way communication.

\section{Progression of 'man-in-the-middle-attack'}

Effective MITM execution has two distinct stages: interception and decryption; which involves being within physical closeness to the intended target, and another that exclusive involves malware, known as a man-in-the-browser (MITB) attack. With a conventional MITM attack, the attacker needs access to an unsecured, or ineffectively anchored Wi-Fi switch (Li et al., 2017; Rahim, 2017; Fei et al., 2018; Howell et al., 2018; Sun et al., 2018). These sorts of associations are by and large found out in the open territories with free Wi-Fi hotspots, and even in a few people's homes. An attacker will check the switch using code looking for particular shortcomings, for example, default or poor secret key utilize, or security gaps because of the poor arrangement of the switch. Once the attacker has discovered the powerlessness, they will then insert their instruments in the middle of the clients' PC and the sites the client visits. A fresher variation of this attack has been gaining fame with cybercriminals because of its simplicity of execution. With a man-in-the-browser attack, every one of an attacker needs are an approach to inject malware into the PC, which will then install itself into the browser without the clients' learning and will then record the information that is being sent between the victim and particular focused on sites, for example, financial institutions, that are coded into the malware. Once the malware has gathered the particular information it was modified to gather, it then transmits that information back to the attacker.

\subsection{Interceoption}

The initial step intercepts client activity through the attacker's system before it achieves its intended destination. The most well-known (and easiest) method for doing this is an inactive attack in which an attacker makes free/open wifi hotspots; accessible to general society. Commonly named in a way that relates to their area, they aren't watchword secured. Once a casualty interfaces with such a hotspot, the attacker gains full permeability to any online information trade. Attackers wishing to adopt a more dynamic strategy to interception may dispatch one of the following attacks:

- IP spoofing involves an attacker disguising himself as an application by altering parcel headers in an ip address. Accordingly, clients attempting to get to a url associated with the application are sent to the attacker's site ('man in the middle (mitm) attack' (incapsula co.), 2016)

- ARP spoofing is the way toward linking an attacker's mac address with the ip address of a legitimate user on a local area network using fake arp messages. Subsequently, information sent by the client to the host ip deliver is instead transmitted to the attacker (Meyer \& Wetzel, 2004; Kish, 2006; Hypponen \& Haataja, 2007; Ouafi et al., 2008; Callegati et al., 2009; Joshi et al., 2009; Desmedt, 2011) 
- DNS spoofing, otherwise called DNS store poisoning, involves infiltrating a DNS server and altering a site's address record. Accordingly, clients attempting to get to the site are sent by the adjusted dns record to the attacker's site (Ouafi et al., 2008; Joshi et al., 2009; Khader et al., 2015; Howell et al., 2018; Sun et al., 2018; Usman et al., 2018; Valluri, 2018; Kuo et al., 2018; Saif et al., 2018; 'man in the middle (mitm) attack' (incapsula co.)).

\subsection{Decryption}

After an interception, any two-way SSL movement should be unscrambled without alerting the client or application. Various strategies exist to accomplish this:

- HTTPS spoofing sends an imposter endorsement to the victim's browser once the initial association demand for a safe site is made ('Man-in-the-middle attack' (Wikipedia)). It holds an advanced thumbprint related with the bargained application, which the browser confirms according to an existing rundown of confided in destinations. The attacker is then ready to get to any information entered by the casualty before it's passed to the application.

- SSL BEAST (browser abuse against SSL/TLS) focuses on a TLS variant 1.0 helplessness in SSL. Here, the casualty's PC is infected with pernicious JavaScript that intercepts scrambled treats sent by a web application. Then the application's figure square chaining (CBC) is endangered in order to decode its treats and authentication tokens ('man-in-the-middle-attack-mitm' (Techpedia); "man-in-the-middleattack" (Rapid Web Ser.); 'What is a Man In The Middle attack?' (Symantec Corp.), Norton Security Blog,; 'What is UMTS?' (Tech Target Web), Blog Post)

- SSL hijacking happens when an attacker passes produced authentication keys to both the client and application during a TCP handshake. This sets up what seems, by all accounts, to be a safe association when, actually, the man in the middle controls the whole session (K. Ouafi et al., 2008; Y. Desmedt, 2011; 'Man-in-the-middle attack' (Wikipedia); 'Flaw in Windows DNS client exposed millions of users to hacking' (SC Mag. UK), News Article)

- SSL stripping minimizes an HTTPS association with HTTP by intercepting the TLS authentication sent from the application to the client. The attacker sends a decoded form of the application's site to the client while maintaining the anchored session with the application. In the meantime, the client's whole session is noticeable to the attacker (Conti et al., 2016; Li et al., 2017; Rahim, 2017; Fei et al., 2018; Howell et al., 2018; Sun et al., 2018; Usman et al., 2018; Valluri, 2018).

\section{MITM: What and how?}

'Man-in-the-middle-attack' also known/abbreviated as MIM, MiM, MitM or MITMA is a type of cryptographic attack over a communication channel by a malicious third party where he/she takes over a confidential/personal communication channel between two or legitimate communicative points or parties. In this cyber attack, the attacker can control (read, modify, intercept, change or replace) the communication traffic between victims. But by using MITM protocol the unauthenticated attacker leaves no clues/traces of his interception of this cybercrime, in short words the attacker remains invisible to the victims.

It needs a communication channel to make a MITM attack. The most used communication channels of MITM attack are namely GSM, UMTS, Long-Term Evolution (LTE), Bluetooth, Near Field Communication (NFC), Radio Frequency and Wi-Fi. The first recorded MITM attack was planned in the time of WW-II for intercepting German Military's radio communication and was done by the Royal British Intelligence (also known as MI-6) (Kozaczuk, 1984). In normal sense, there are three most possible compromises, namely Confidentiality, Integrity, and Availability; which is aimed at my MITM attack. Most of the MITM attacks now days are done in social media, because of the extensive use of human communication are done using social media (Facebook, Twitter, Yahoo Messenger and etc. (Hudaib, 2014) Decoding a MITM attack is a long process, basically this is done using three ways, namely 1) Based on 
impersonation methods of cyber decoding, 2) Based on Telecommunication addressing techniques and lastly 3) Based on GPS locating method of attacker and victims both (Conti et al., 2016).

\section{Present status of MITM attacks}

Nowadays, most of the MITM attacks are performed using communication layers. Open System Intercommunication (OSI) and GSM networks are the most affected communication channels by MITM attacks. Table-1 shows types of MITM attacks on different OSI and Cellular service networks ('Man-inthe-middle attack' (Wikipedia); 'man-middle-attack' (CA Tech); 'man-in-the-middle-attack-mitm' (Techpedia); “man-in-the-middle-attack” (Rapid Web Ser.); 'What is a Man In The Middle attack?' (Symantec Corp.), Norton Security Blog); 'What is UMTS?' (Tech Target Web), Blog Post; 'Flaw in Windows DNS client exposed millions of users to hacking' (SC Mag. UK), News Article; Fatima, 2011; Kozaczuk, 1984; Hudaib, 2014; Conti et al., 2016).

Table 1

MITM attacks on different communication channels

\begin{tabular}{ccc} 
& & MITM Types \\
\hline OSI Layers & Data Links & ARP spoofing type \\
& Presentation & SSL decryption, CA decryption \\
& Transport and Networking & IP spoofing \\
Cellular Networks & Applications & DHCP spoofing, BGP type, DNS spoofing \\
\hline & GSM & FBS type \\
\hline
\end{tabular}

In Table 1, we list MITM attacks across OSI layers and cellular networks. Each layer enforces different approaches to provide security. Nevertheless, neither of them is free from MITM attacks. Ornaghi et al. 2003, at a European conference, was the first to present a security system-based tracking location of the attacker and victim. He classified MITM attacks in three distinct categories: a) LAN (Local Area Network) tracking, b) LAN to Remote Network tracking and c) Remote Network track. The authors also take into consideration that STP mangling is a closed type of MITM as the attacker can only manage to decode the unmanaged traffic between two clients.

\subsection{Spoofing: Most common MITM}

Spoofing an impersonation technique which is originated from 'spying'. In the middle century, European spies used to hear secret conversation by impersonating him/her to the communicative party. The same method is applied in modern cryptographic spoofing, as the attacker intercepts a confidential/personal communication between two hosts and controls over transferring data, while the hosts are not being aware of the unauthenticated attacker. Some research papers ('Flaw in Windows DNS client exposed millions of users to hacking' (SC Mag. UK), News Article; 'What is UMTS?' (Tech Target Web), Blog Post); 'What is a Man in The Middle attack?' (Symantec Corp.), Norton Security Blog; "man-in-the-middleattack" (Rapid Web Ser.), Blog Post; 'man-in-the-middle-attack-mitm' (Techpedia); 'man-middle-attack' (CA Tech.); 'Man-in-the-middle attack' (Wikipedia); 'MAN IN THE MIDDLE (MITM) ATTACK' (Incapsula Co.); Saif et al., 2018; Kuo et al., 2018; Valluri, 2018; Usman et al., 2018; Senie \& Ferguson, 1998; Humphreys et al., 2008; Scott, 2001; Schuckers, 2002) describe spoofing as the first step of executing MITM, not being the total of a MITM attack; while some other deliciated research papers claim spoofing as a whole MITM process. In this paper, we will consider it as a spoofing based MITM or spoofing attack. When a party wants to communicate with other parties over a cryptographic network then if their network is same with an unknown MAC address then the server broadcasts an address resolution protocol (also abbreviated as ARP) request to all hosts under the same network connection. The client with the announced Internet Protocol is only expected to make a reply including his/her MAC (Media Access Control) address. However, when ARP cache is managed in a dynamic 
mode, cache entries can be easily fabricated by forged ARP messages, since proper authentication mechanism is missing (Oh et al., 2012). In the meantime, the communicating medium saves the IP to MAC entry in its local cache, so the next time communication can be speeded up, by avoiding the broadcasts.

Address Resolution Protocol has no states thus it provides very few securities to the caching system. Some top-notch researches referring from Ataullah and Chauhan (2012), Altunbasak et al. (2004), Subashini and Kavitha (2011), Alabady (2009), Caceres and Padmanabhan (1998), Ford (2005), Pansa and Chomsiri (2008), Chomsiri (2008), Salim et al. (2012), Demuth and Leitner (2005) shows the stateof-art (SoA) of using those security weaknesses for conducting a perfect MITM attack. Suppose, we have next network: the attacker ' $\mathrm{X}$ ' $(\mathrm{IP}=10.0 . \mathrm{x} . \mathrm{X} 3, \mathrm{MAC}=\mathrm{EE}: \mathrm{EE}: \mathrm{EE}: \mathrm{EE}: \mathrm{EE}: \mathrm{X} 3)$, victim 'A' (IP=10.0.X.X1, $\mathrm{MAC}=\mathrm{AA}: \mathrm{AA}: \mathrm{AA}: \mathrm{AA}: \mathrm{AA}: \mathrm{X} 1)$, and victim 'B' (IP = 10.0.x.x2, MAC = BB:BB:BB:BB:BB:X2). The next steps for a perfect spoofing based on ARP are shown below:

1)' $X$ ' sends an ARP Reply message to 'A', which says that IP: 10.0.X.x3 has MAC address: EE:EE:EE:EE:EE:X3. This message will update 'A'”s ARP table.

2)' $\mathrm{X}$ ' also sends an ARP Reply message to ' $\mathrm{B}$ ', which says that IP: 10.0.X.X2 has MAC address: EE:EE:EE:EE:EE:X3. This message will update 'B''s ARP table.

3)When ' $A$ ' wants to send a message to ' $B$ ', it will go to ' $X$ 's MAC address EE:EE:EE:EE:EE:X3, instead of 'B''s BB:BB:BB:BB:BB:X2.

4) When 'B' wants to send a message to 'A', it will also go to ' $X$ '.

Schematic regarding the example stated above is given in Fig. 2.

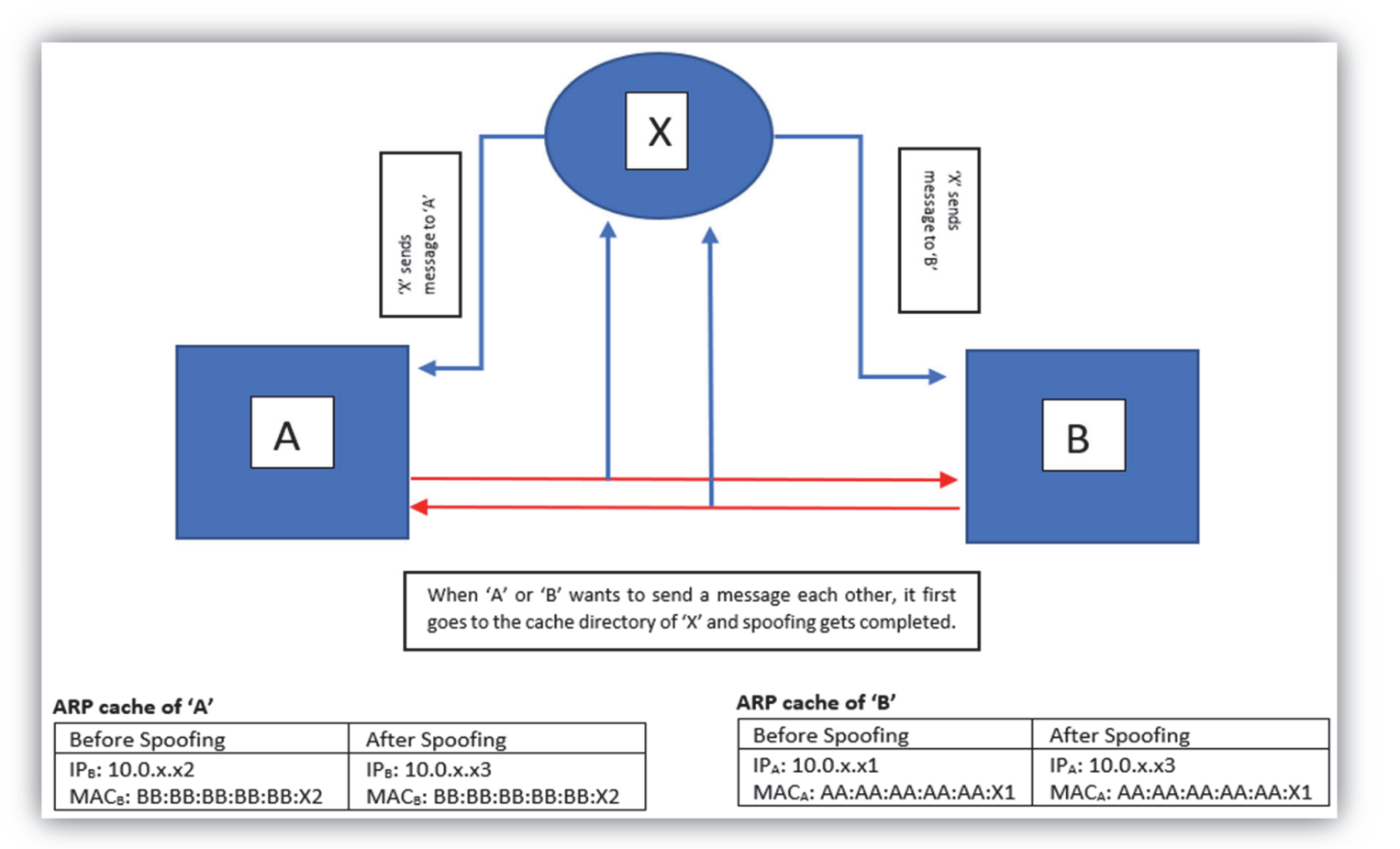

Fig. 2. Spoofing method between two clients

There are many well-researched works of literature where spoofing defending system is discussed. Among them T. Demuth et al., 2005, D. Pansa et al., 2008, Z. Trabelsi et al., 2007 and R. Philip et al., 2007 are mostly considerable (D. Pansa and T. Chomsiri, 2008; T. Demuth and A. Leitner, 2005; Z. 
Trabelsi and W. El-Hajj, 2007; R. Philip et al., 2007). They introduced various well-researched techniques to prevent spoofing and make secure communication over LAN. But those Literature doesn't concern about wireless methods of communications. Table 2 below shows a typical comparison between spoofing prevention techniques:

Table 2

Comparison of various types of spoofing prevention

\begin{tabular}{|c|c|c|c|}
\hline References & Medium of Communication & Protocol & Concerns \\
\hline Demuth et al., 2005 & Server Based Communication & ARP & Can't work for wireless communications. \\
\hline Pansa et al., 2008 & Server Based/ Host Based & ARP, DHCP & Compatible for DoS, DHCP but has a single point of failure. \\
\hline Trabelsi et al., 2007 & Host Based & ARP & Level of importance of each host is very difficult to decide. \\
\hline Philip et al., 2007 & Host Based & ARP & Works only with Linksys routers. Static IP not supported. \\
\hline Oh et al., 2012 & Cryptographic/ Host Based & UDP/ARP & For UDP, authentication is a must need. \\
\hline Komori et al., 2002 & $\begin{array}{l}\text { SYMMETRIC/PRIVATE-KEY } \\
\text { CRYPTOGRAPHY }\end{array}$ & DHCP & $\begin{array}{l}\text { Legitimate hosts must register in advance, adds additional } \\
\text { message flow, hard to manage for large number of hosts. }\end{array}$ \\
\hline Ju et al., 2007 & $\begin{array}{l}\text { SYMMETRIC/PRIVATE-KEY } \\
\text { CRYPTOGRAPHY, RFC }\end{array}$ & DCHP, DHCP & $\begin{array}{l}\text { The authors did not describe how the random value (the } \\
\text { number, which used by the server and client to compute the } \\
\text { session key) is determined. }\end{array}$ \\
\hline Duan et al., 2006 & Router Based & IP, ARP & $\begin{array}{l}\text { Filtering-on-path method can't ensure a secure } \\
\text { communication. }\end{array}$ \\
\hline Andersen et al., 2008 & Router/ Host Based & IP, DHCP & $\begin{array}{l}\text { This system is considered as the highest secured } \\
\text { communication. But not so user friendly. }\end{array}$ \\
\hline
\end{tabular}

\section{MITM on GSM: A threat to phone communication security}

In the early 90's, the European Telecommunications Standards Institute introduced GSM as a second generation $(2 \mathrm{G})$ telecommunication standard. Today, according to the mobility report (SAMSUNG ELECTRONICS SUSTAINABILITY REPORT), GSM covers more than $90 \%$ of the world population. There are two basic types of services offered through GSM: telephony and data bearer. The GSM architecture consists of Mobile Stations (MSs) and Base Terminal Stations (BTS), which communicate with each other through radio links. Each BTS connects to the Base Station Controller (BSC). BSC links to the Mobile Switching Center (MSC), which is responsible for routing signals to and from fixed networks (Z. Su et al., 2018). Home Location Register (HLR) and the Visitor Location Register (VLR) are the two major databases for each mobile service provider in the GSM architecture. Fig. 3 shows a schematic of GSM architecture. Each of GSM subscribers has the secret key, which is stored in the Subscriber Identity Module (SIM) card of the MS. The Authentication Center (AUC) has a secret key, which is shared with the subscriber and AUC. AUC generates a set of security parameters for execution of encryption and authentication.

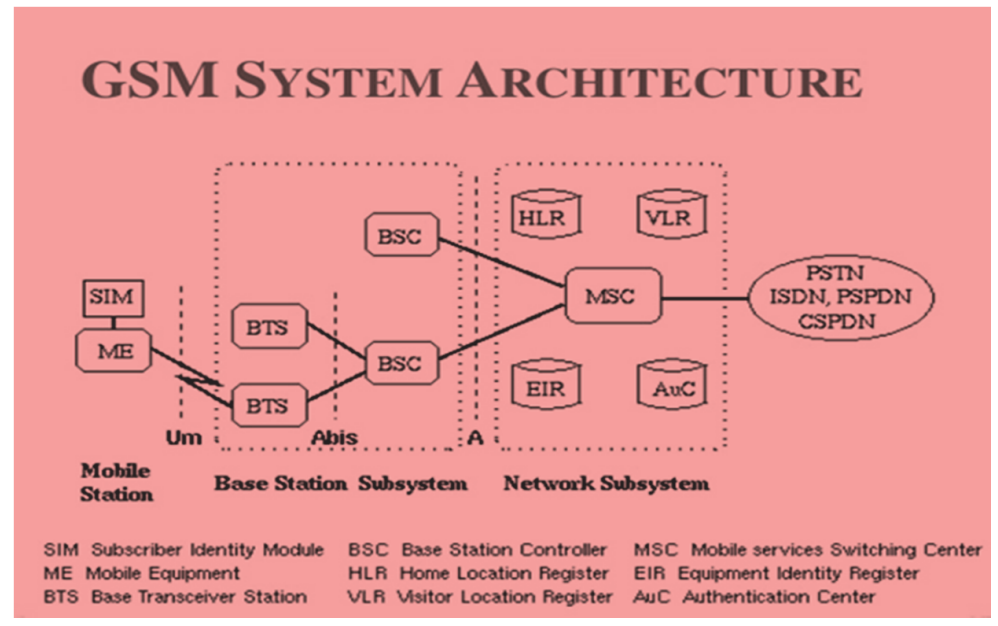

Fig. 3. GSM Architecture (Kurose, 2005)

The main idea behind the attack is to impersonate same mobile network code as the legitimate GSM network to false BTS (or IMSI Cather (Hardin, 2018)) and convince the victim that this station is the valid one. Let us consider the next example: network consists of the Legitimate MS, Legitimate BTS, 
False BTS, and False MS. Attacker's network is a combination of the False BTS and False MS. While in standby mode the MS connects to the best received BTS. Therefore, False BTS should be more powerful than the original one, or closer to the target. If the victim is already connected, then the attacker requires to drawn any present real stations. The algorithm of the FBS-based MITM attack on GSM is the following:

1) Attacker sets-up connection between False BTS and Legitimate MS.

2) False MS impersonates the victim's MS to the real network by resending the identity information, which was received from the step 1 .

3) Victim's MS sends its authentication information and cipher-suites to the False BTS.

4) Attacker forwards message from step 3 to the Legitimate BTS, with changed authentication abilities of the MS to do not support encryption (A5/0 algorithm (Su X. et al. 2018)), or to weak encryption algorithm (e.g., A5/2).

5) Legitimate MS and Legitimate BTS exchange authentication challenge (RAND), and authentication response (SRES), attacker forwards them.

Fig. 4 below shows a graphical representation of the example stated above.

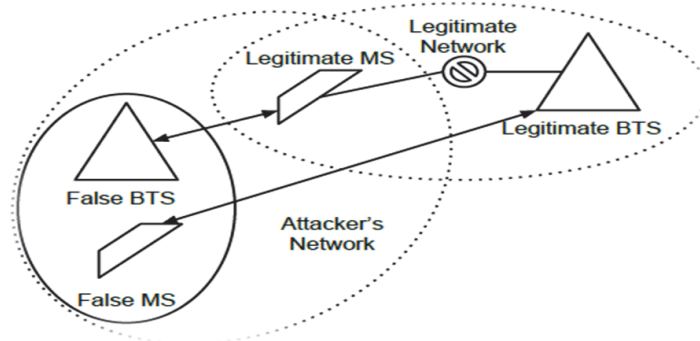

Fig. 4. MITM in GSM network

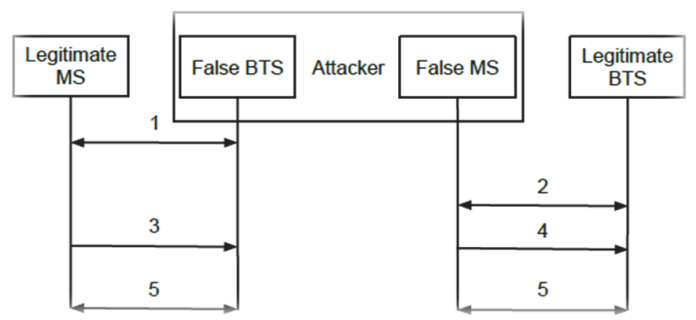

Fig. 5. MITM on GSM network via FBS method

Finally, the authentication is finished. All following messages between the victim and real network are going through attacker's entities, with encryption specified by an attacker, or no encryption at all. This manipulation is possible since GSM does not provide the data integrity (Chen et al., 2007), as a result, the attacker can catch, modify, and resend messages. At the designing phase of the GSM protocol, FBS seemed impractical due to costly required equipment, but currently, this kind of attack is completely applicable since costs decreased (Feher et al., 2018). Paik et. al. (2010); besides describing GSM security concerns, pointed out that nowadays attackers are better equipped. Among the reasons we can identify opensource projects (e.g., Open BTS (Burgess \& Samra, 2008)) and low-cost hardware (e.g., Ettus Research (A. N. I. C. Ettus Research. Ettus research - the leader in software-defined radio (SDR))). In particular, an attacker can build its own false BTS for less than \$1000. An algorithm of FBD based MITM attack on GSM network is given below in Fig. 5. Table 3 discusses various FBS based MITM attacks prevention approaches and different attacks with regarding references.

Table 3

FBS based MITM attacks preventions

\begin{tabular}{|c|c|c|c|c|c|}
\hline Preventions & Ou et al., 2010 & Huang et al., 2011 & Hwang et al., 2014 & $\begin{array}{l}\text { Saxena and } \\
\text { Chaudhari, } 2014\end{array}$ & $\begin{array}{ll}\text { Saxena } & \text { and } \\
\text { Chaudhari, 2014 } & \end{array}$ \\
\hline MITM attacks & Yes & Yes & Yes & Yes & Yes \\
\hline Replay attacks & Yes & Yes & Yes & Yes & Yes \\
\hline $\begin{array}{l}\text { Active attack in } \\
\text { unauthorized network }\end{array}$ & Yes & Yes & Yes & Yes & Yes \\
\hline Redirection & Yes & Yes & Yes & Yes & Partially \\
\hline DoS attack & No & Partially & Yes & No & Yes \\
\hline
\end{tabular}




\section{Statistical analysis of MITM attack}

For statistical analysis of the MiM attacks, we refer to the usual finite lattice of security levels, $\left(\mathcal{S}, \sqsubseteq_{\mathcal{S}}, \Pi_{\mathcal{S}}, \sqcup_{\mathcal{S}}, \mathrm{T}_{\mathcal{S}}, \perp_{\mathcal{S}}\right)$ and based on it define $\zeta: \mathbb{N} \rightarrow \mathcal{S}$ as a mapping from names to their security levels. Now, we can define the name integrity property as follows.

Property [Name integrity]

We say that a name, $x$, has the integrity property with respect to a $\phi_{A}$ environment if

$$
\forall n \in \text { value }_{o f\left(\phi_{A}(x)\right)}: \zeta(x) \sqsubseteq \zeta(n)
$$

The predicate integrity $\left(x, \phi_{A}\right)$ indicates that $x$ upholds the above property with respect to $\phi_{A}$. A MITM attack is defined as an attack in which the intruder is capable of breaching the integrity of names of two processes.

\section{Property [Man-in-the-Middle Attack]}

A context, $C$ (a process with a hole) succeeds in launching a MiM attack on two processes, $P$ and $Q$, if the result of the abstract interpretation, $A\left(\mid C(P|Q|)\{||\} \perp_{D_{\perp}}=\phi_{A}\right.$ proves that $\exists_{x} \in b n(P), y \in$ $\operatorname{bn}(Q): \neg\left(\operatorname{integrity}\left(x, \phi_{A}\right) \vee\right.$ integrity $\left.\left(y, \phi_{A}\right)\right)$.

\section{Preventing MITM}

Blocking MITM attacks requires a few down to earth ventures with respect to clients, and additionally a combination of encryption and check techniques for applications. For clients, this implies:

- Avoiding WiFi associations that aren't password encrypted.

- Paying consideration regarding browser warnings reporting a site as being unsecured.

- Immediately logging out of a protected application when it's not in utilize.

- Not using open systems (e.g., cafés, lodgings) when conducting sensitive financial exchanges.

For site administrators, secure correspondence conventions, including TLS and HTTPS, help relieve spoofing attacks by vigorously encrypting and authenticating transmitted information (Fatima, 2011). Doing so keeps the interception of site activity and hinders the decoding of delicate information, for example, authentication tokens. It is viewed as best practice for applications to utilize SSL/TLS to anchor each page of their site and not only the pages that expect clients to sign in. Doing so helps diminishes the possibility of an attacker stealing session treats from a client browsing on an unsecured segment of a site while signed in.

To counter MITM, Antivirus frameworks furnishes its clients with a streamlined end-to-end SSL/TLS encryption, as a component of its suite of security administrations ('Man-in-the-middle attack' (Wikipedia); 'man-middle-attack' (CA Tech.); 'man-in-the-middle-attack-mitm' (Techpedia); 'man-in-the-middle-attack" (Rapid Web Ser.), Blog Post; 'What is a Man In The Middle attack?' (Symantec Corp.), Norton Security Blog; 'What is UMTS?' (Tech Target Web), Blog Post; 'Flaw in Windows DNS client exposed millions of users to hacking' (SC Mag. UK), News Article). Facilitated on well-known Antispam administrations content conveyance arrange $(\mathrm{CDN})$, the authentications are ideally executed to forestall SSL/TLS compromising attacks, for example, minimize attacks (e.g. SSL stripping), and to guarantee compliance with most recent PCI DSS demands. Offered as a managed benefit, SSL/TLS arrangement is stayed up with the latest maintained by an expert security, both to stay aware of compliance demands and to counter emerging dangers (e.g. Heartbleed) ('What is a Man In The Middle attack?' (Symantec Corp.), Norton Security Blog). Finally, with Antivirus dashboards, the client can likewise design HTTP Strict Transport Security (HSTS) arrangements to implement the utilization of SSL/TLS 
security over different subdomains. This furthers secure site and web application from convention minimize attacks and treat hijacking endeavors. Table 4 gives a typical review of different types of prevention methodologies.

Table 4

Various MITM prevention Mechanisms studied from

\begin{tabular}{|c|c|c|c|c|c|c|}
\hline \multirow{2}{*}{$\begin{array}{l} \\
\text { OSI }\end{array}$} & \multirow{2}{*}{ Application } & \multicolumn{5}{|l|}{ Approaches } \\
\hline & & Detection & $\begin{array}{l}\text { Cryptography } \\
\text { BGP:(Mitseva et al., } \\
\text { 2018; Preneel and } \\
\text { Frederik Vercauteren, } \\
\text { 2015; Haya Shulman, } \\
\text { 2018; Flores et al., } \\
\text { 2018) }\end{array}$ & \multirow[b]{2}{*}{$\begin{array}{l}\text { Voting } \\
\text { DNS:('man-in-the-middle- } \\
\text { attack'(CA.Tech.)), (Duan } \\
\text { et al., 2006; Andersen et } \\
\text { al., 2008; SAMSUNG } \\
\text { ELECTRONICS } \\
\text { SUSTAINABILITY } \\
\text { REPORT, 2017; Su et al., } \\
\text { 2018; Hardin 2018; Su Xin } \\
\text { et al., 2005), (Flores } \text { et al., } \\
\text { 2018; Fernàndez-València } \\
\text { et al., 2018; Hanna et al., } \\
\text { 2018) }\end{array}$} & \multirow[b]{2}{*}{ 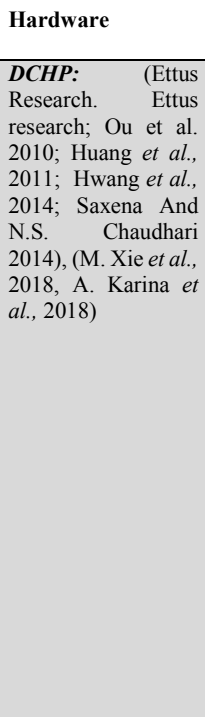 } & $\begin{array}{l}\text { Other } \\
\text { BGP: (Naqash et al., } \\
\text { 2012; Kaminsky, 2008; } \\
\text { Lindell, 2018; Xiang, } \\
\text { 2018; Zhang, 2018) }\end{array}$ \\
\hline OSI & Application & $\begin{array}{l}\text { BGP: (Mayer and } \\
\text { Susanne, } 2014 ; \\
\text { Hypponen and Keijo, } \\
\text { 2007; Ouafi et al., 2008; } \\
\text { Callegati et al., 2009; } \\
\text { Joshi et al., 2009; Yvo } \\
\text { Desmedt, 2011; } \\
\text { Sounthiraraj et al., } \\
\text { 2014; Khader and Lai, } \\
\text { 2015; Yu-Chih Tung et } \\
\text { al., 2016; Wallace and } \\
\text { Miller, 2017; Conti et } \\
\text { al., 2016; Li } \text { et al., 2017; } \\
\text { Rahim, 2017; Yang- } \\
\text { Yang Fei et al., 2018; } \\
\text { Howell et al., 2018; Da- } \\
\text { Zhi Sun et al., 2018; } \\
\text { Usman et al., 2018; } \\
\text { Valluri 2018; En-Chun } \\
\text { Kuo et al., 2018; Saif } \text { et } \\
\text { al., 2018), Stiansen, } \\
\text { 2018; Chaz \& Kim- } \\
\text { Kwang Raymond Choo, } \\
\text { 2018; T. Stiansen et al., } \\
\text { 2018) } \\
\text { DNS: (Yong Wan Ju et } \\
\text { al., 2007; Chopra and } \\
\text { MichaelKaufman, 2014; } \\
\text { Naqash et al. 2012; } \\
\text { Kaminsky 2008; Lindell } \\
\text { 2018; Li Xiang et al., } \\
\text { 2018; D. Zhang, Yuezhi } \\
\text { Zhou and Yaoxue } \\
\text { Zhang, 2018) }\end{array}$ & $\begin{array}{l}\text { BGP:(Mitseva et al., } \\
\text { 2018; Preneel and } \\
\text { Frederik Vercauteren, } \\
\text { 2015; Haya Shulman, } \\
\text { 2018; Flores et al., } \\
\text { 2018) } \\
\text { DCHP: (Ou et al., } \\
\text { 2010; Huang et al., } \\
\text { 2017; Hwang and } \\
\text { Prosanta Gope, 2014; } \\
\text { Saxena and } \\
\text { Chaudhari, 2014 (1 \& } \\
\text { 2)) }\end{array}$ & & & $\begin{array}{l}\text { BGP: (Naqash et al., } \\
\text { 2012; Kaminsky, 2008; } \\
\text { Lindell, 2018; Xiang, } \\
\text { 2018; Zhang, 2018) } \\
\text { DNS: (Stiansen et al., } \\
\text { 2018; Mitseva et al., } \\
\text { 2018; Preneel and } \\
\text { Frederik Vercauteren, } \\
\text { 2015) }\end{array}$ \\
\hline & Presentation & $\begin{array}{l}\text { SSL/TLS: (M. Ulrike } \\
\text { and Susanne Wetzel, } \\
\text { 2004; Laszlo and Kish, } \\
\text { 2006; Hypponen and } \\
\text { Haataja, 2007; Ouafi et } \\
\text { al., 2008; Callegati et } \\
\text { al., 2009; Joshi et al., } \\
\text { 2009; Yvo Desmedt, } \\
\text { 2011; Sounthiraraj et } \\
\text { al., 2014; Khader and } \\
\text { David Lai, 2015; Yu- } \\
\text { Chih Tung et al., 2016; } \\
\text { Wallace and Miller, } \\
\text { 2018; Conti et al., 2016; } \\
\text { Xiaohong Li et al., } \\
\text { 2017), (Logan Scott, } \\
\text { 2001) }\end{array}$ & $\begin{array}{l}\text { SSL/TLS: (Wallace } \\
\text { and Miller, 2016; } \\
\text { Conti et al., 2016; } \\
\text { Stiansen, 2018; Chaz } \\
\text { and Kim-Kwang } \\
\text { Raymond Choo, } \\
\text { 2018; Stiansen et al., } \\
\text { 2018; Li et al., 2017), } \\
\text { (Karina et al., 2015; } \\
\text { Nath et al., 2018; } \\
\text { Hossain et al., 2018; } \\
\text { Sinor, 2018) }\end{array}$ & $\begin{array}{l}\text { SSL/TLS: (L. Scott, 2001; } \\
\text { M. Oh et al., 2012) }\end{array}$ & - & $\begin{array}{l}\text { SSL/TLS: (Sinor, 2018; } \\
\text { Gunawan, 2018) }\end{array}$ \\
\hline & Transport & - & $\begin{array}{l}\text { IP: (Ulrike Meyer } \\
\text { and Susanne Wetzel, } \\
\text { 2004; Laszlo B. Kish, } \\
\text { 2006; K. Hypponen } \\
\text { and Keijo MJ } \\
\text { Haataja, 2007; K. } \\
\text { Ouafi et al., 2008; F. } \\
\text { Callegati } \text { et al., 2009; } \\
\text { Y. Joshi et al., 2009; } \\
\text { Yvo Desmedt, 2011; } \\
\text { D. Sounthiraraj et al., } \\
\text { 2014; A. S. Khader } \\
\text { and David Lai, 2015; } \\
\text { Yu-Chih Tung et al., } \\
\text { 2016; B. M. Wallace } \\
\text { and W. S. Miller, } \\
\text { 2016; M. Conti et al., } \\
\text { 2016; X. Li et al., } \\
\text { 2017; R. Rahim, } \\
\text { 2017; Yang-Yang Fei } \\
\text { et al., 2018; C. }\end{array}$ & - & - & $\begin{array}{l}\text { IP: (Huang et al., 2018; } \\
\text { Goodman et al., 2018; } \\
\text { Wang et al., 2018) }\end{array}$ \\
\hline
\end{tabular}




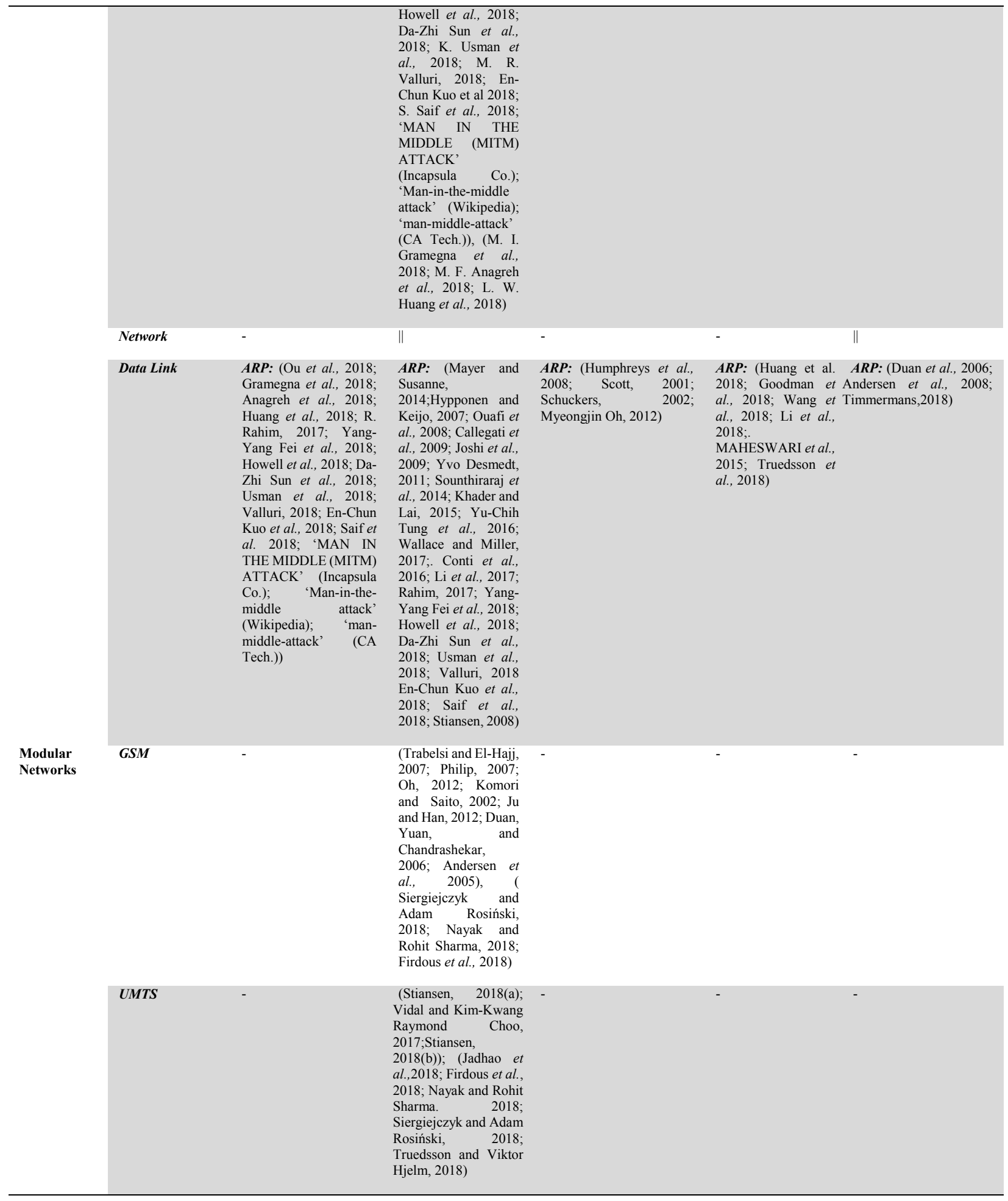

\section{Conclusion}

The MITMs interrupt interchanges between two frameworks, and this phenomenon takes place when the attacker is responsible for a switch along typical point of movement. The attacker in all cases is situated on a similar communicated domain as the victim stands. Indeed, in a HTTP exchange, a TCP protocol exists among the customer and the server. The attacker divides the TCP protocol into two connections one between the victim and the attacker and the other between the attacker and the server. On intercepting 
the TCP protocol, the attacker goes about as an intermediary reading, altering and inserting information in intercepted correspondence. In an unsecured connection (e.g. HTTP protocol), the communication of two users can be hacked by an intruder without any difficulties. In a HTTPS connection, a single TCP protocol is attained by building two independent SSL connections. A MITM attack exploits the shortcoming in arrange correspondence convention, convincing the casualty to course movement through the attacker instead of ordinary switch and is by and large alluded to as ARP spoofing. This unethical phenomenon can affect a country's economy and may be a reason of instability between nations by stealing/modifying classified/secret defense sector data/information. So, this unethical phenomenon has to be prevented, and the necessary measures should be taken for ending. Although the paper did not focus on extensive analysis for future research directions of MITM, but a good understanding about MITM and the technologies for preventing MITM like Li-Fi were discussed briefly.

\section{Acknowledgement}

The authors are grateful to the Dept. of Mechanical Engineering and Dept. of Computer Science and Engineering, RUET for providing technical and financial support to conduct this survey research.

\section{References}

A. N. I. C. Ettus Research. Ettus research - the leader in software defined radio (sdr). [Online]. Available: http://www.ettus.com

Alabady, S. (2009). Design and Implementation of a Network Security Model for Cooperative Network. Int. Arab J. e-Technol., 1(2), 26-36.

Altunbasak, H., Krasser, S., Owen, H., Sokol, J., \& Grimminger, J. (2004, November). Addressing the weak link between layer 2 and layer 3 in the Internet architecture. In Local Computer Networks, 2004. 29th Annual IEEE International Conference on (pp. 417-418). IEEE.

Anagreh, M. F., Hilal, A. M., \& Ahmed, T. M. (2018). Encrypted Fingerprint into VoIP Systems using Cryptographic Key Generated by Minutiae Points. INTERNATIONAL JOURNAL OF ADVANCED COMPUTER SCIENCE AND APPLICATIONS, 9(1), 151-154.

Andersen, D. G., Balakrishnan, H., Feamster, N., Koponen, T., Moon, D., \& Shenker, S. (2008, August). Accountable internet protocol (aip). In ACM SIGCOMM Computer Communication Review (Vol. 38, No. 4, pp. 339350). ACM.

Ataullah, M., \& Chauhan, N. (2012, March). ES-ARP: an efficient and secure address resolution protocol. In Electrical, Electronics and Computer Science (SCEECS), 2012 IEEE Students' Conference on (pp. 1-5). IEEE

Burgess, D. A., \& Samra, H. S. (2008). The openbts project. Report available at http://openbts. sourceforge. net, http://openBTS. org.

Caceres, R., \& Padmanabhan, V. N. (1998). Fast and scalable wireless handoffs in support of mobile Internet audio. Mobile Networks and Applications, 3(4), 351-363

Callegati, F., Cerroni, W., \& Ramilli, M. (2009). Man-in-the-Middle Attack to the HTTPS Protocol. IEEE Security \& Privacy, 7(1), 78-81.

Chen, Z., Guo, S., Zheng, K., \& Yang, Y. (2007, September). Modeling of man-in-the-middle attack in the wireless networks. In Wireless Communications, Networking and Mobile Computing, 2007. WiCom 2007. International Conference on(pp. 2255-2258). IEEE.

Chomsiri, T. (2008, November). Sniffing packets on LAN without ARP spoofing. In Third 2008 International Conference on Convergence and Hybrid Information Technology (pp. 472-477). IEEE.

Chopra, A., \& Kaufman, M. (2014). Man In the Middle (MITM) DNS Spoofing Explained.

Conti, M., Dragoni, N., \& Lesyk, V. (2016). A survey of man in the middle attacks. IEEE Communications Surveys \& Tutorials, 18(3), 2027-2051

Conti, M., Dragoni, N., \& Lesyk, V. (2016). A survey of man in the middle attacks. IEEE Communications Surveys \& Tutorials, 18(3), 2027-2051.

Demuth, T., \& Leitner, A. (2005). ARP spoofing and poisoning: Traffic tricks. Linux magazine, 56, 26-31.

Desmedt, Y. (2011). Man-in-the-middle attack. In Encyclopedia of cryptography and security (pp. 759-759). Springer, Boston,

Duan, Z., Yuan, X., \& Chandrashekar, J. (2006, March). Constructing Inter-Domain Packet Filters to Control IP Spoofing Based on BGP Updates. In INFOCOM.

Fatima, A. (2011). E-Banking Security Issues-Is There A Solution in Biometrics?. Journal of Internet Banking and Commerce, 16(2), 1 
Feher, B., Sidi, L., Shabtai, A., Puzis, R., \& Marozas, L. (2018). WebRTC security measures and weaknesses. International Journal of Internet Technology and Secured Transactions, 8(1), 78-102.

Fei, Y. Y., Meng, X. D., Gao, M., Wang, H., \& Ma, Z. (2018). Quantum man-in-the-middle attack on the calibration process of quantum key distribution. Scientific reports, 8(1), 4283.

Fernàndez-València, R., Caubet, J., \& Vila, A. (2018). Cryptography Working Group Introduction to Blockchain Technology.

Firdous, G. S., \& Kumar, R. S. (2018). SUPPORT DATA ACCESS ORGANIZE MECHANISM OF RELEASE ENCRYPTION PRIVACY AND SECURITY PROTECTION. IJITR, 6(2), 7937-7939.

'Flaw in Windows DNS client exposed millions of users to hacking' (SC Mag. UK), News Article, 2017, Retrieved from: https://www.scmagazineuk.com/flaw-in-windows-dns-client-exposed-millions-of-users-to-hacking/article/699416/

Flores, M., Wenzel, A., Chen, K., \& Kuzmanovic, A. (2018, March). Fury Route: Leveraging CDNs to Remotely Measure Network Distance. In International Conference on Passive and Active Network Measurement (pp. 8799). Springer, Cham.

Ford, M. (2005, January). New internet security and privacy models enabled by ipv6. In Applications and the Internet Workshops, 2005. Saint Workshops 2005. The 2005 Symposium on (pp. 2-5). IEEE.

Goodman, J. L., Maher, H. B., Komanduri, R., \& Raj, R. K. (2018). U.S. Patent Application No. 15/211,272.

Gramegna, M., Berchera, I. R., Kueck, S., Porrovecchio, G., Chunnilall, C. J., Degiovanni, I. P., ... \& Castagna, N. (2018, May). European coordinated metrological effort for quantum cryptography. In Quantum Technologies 2018 (Vol. 10674, p. 106741K). International Society for Optics and Photonics.

Gunawan, D., Sitorus, E. H., Rahmat, R. F., \& Hizriadi, A. (2018, March). SSL/TLS Vulnerability Detection Using Black Box Approach. In Journal of Physics: Conference Series (Vol. 978, No. 1, p. 012121). IOP Publishing.

Hanna, D., Veeraraghavan, P., \& Pardede, E. (2018). PrECast: An Efficient Crypto-Free Solution for BroadcastBased Attacks in IPv4 Networks. Electronics, 7(5), 65.

Hardin, N. V. (2018). UNCOVERING THE SECRECY OF STINGRAYS: What Every Practitioner Needs to Know. Criminal Justice, 32(4), 20-24.

Hasan, S., Awais, M., \& Shah, M. A. (2018, April). Full Disk Encryption: A Comparison on Data Management Attributes. In Proceedings of the 2nd International Conference on Information System and Data Mining (pp. 39-43). ACM.

Hiltunen, M. A., Miluzzo, E., \& Srivastava, A. (2017). U.S. Patent No. 9,818,315. Washington, DC: U.S. Patent and Trademark Office.

Hossain, M. S., Paul, A., Islam, M. H., \& Atiquzzaman, M. (2018). Survey of the Protection Mechanisms to the SSL-based Session Hijacking Attacks. Network Protocols and Algorithms, 10(1), 83-108.

Howell, C., Statica, R., \& Coppa, K. L. (2018). U.S. Patent No. 9,906,506. Washington, DC: U.S. Patent and Trademark Office.

Huang, L. W., Hsu, H. L., \& Kao, H. T. (2018). U.S. Patent No. 9,984,225. Washington, DC: U.S. Patent and Trademark Office.

Huang, Y. L., Shen, C. Y., \& Shieh, S. W. (2011). S-AKA: A provable and secure authentication key agreement protocol for UMTS networks. IEEE Transactions on Vehicular Technology, 60(9), 4509-4519.

Hudaib, A. A. Z. (2014). Comprehensive Social Media Security Analysis \& XKeyscore Espionage Technology. International Journal of Computer Science and Security (IJCSS), 8(4), 97

Humphreys, T. E., Ledvina, B. M., Psiaki, M. L., O'Hanlon, B. W., \& Kintner, P. M. (2008). Assessing the spoofing threat: Development of a portable GPS civilian spoofer. In Radionavigation Laboratory Conference Proceedings.

Hwang, T., \& Gope, P. (2014). Provably secure mutual authentication and key exchange scheme for expeditious mobile communication through synchronously one-time secrets. Wireless personal communications, 77(1), 197-224.

Hypponen, K., \& Haataja, K. M. (2007, September). "Nino" man-in-the-middle attack on bluetooth secure simple pairing. In Internet, 2007. ICI 2007. 3rd IEEE/IFIP International Conference in Central Asia on (pp. 1-5). IEEE.

Jadhao, M. M., Gothe, M. S., \& Nimkarde, M. S. Specific Location Based Privacy protecting Access Control System.

Joshi, Y., Das, D., \& Saha, S. (2009, December). Mitigating man in the middle attack over secure sockets layer. In Internet Multimedia Services Architecture and Applications (IMSAA), 2009 IEEE International Conference on (pp. 1-5). IEEE. 
Ju, H., \& Han, J. (2007). DHCP message authentication with an effective key management. World Academy of Science, Engineering and Technology, International Journal of Electrical, Computer, Energetic, Electronic and Communication Engineering, 1(8), 1199-1202.Z.

Ju, Y. W., Song, K. H., Lee, E. J., \& Shin, Y. T. (2007, February). Cache poisoning detection method for improving security of recursive DNS. In Advanced Communication Technology, The 9th International Conference on (Vol. 3, pp. 1961-1965). IEEE.

Kaminsky, D. (2008). Black ops 2008: It's the end of the cache as we know it. Black Hat USA.

Karina, A., Avila-Pesántez, D., Vaca-Cárdenas, L., Arellano, A., \& Mantilla, C. Towards a Security Model against Denial of Service Attacks for SIP Traffic. World Academy of Science, Engineering and Technology, International Journal of Social, Behavioral, Educational, Economic, Business and Industrial Engineering, 12(1), 8287.

Khader, A. S., \& Lai, D. (2015). Preventing man-in-the-middle attack in Diffie-Hellman key exchange protocol. In 22nd International Conference on Telecommunications: ICT 2015(p. 204). Engineers Australia

Kish, L. B. (2006). Protection against the man-in-the-middle-attack for the Kirchhoff-loop-Johnson (-like)-noise cipher and expansion by voltage-based security. Fluctuation and Noise Letters, 6(01), L57-L63.

Klink, J., \& Little, H. (2018). U.S. Patent Application No. 15/332,057.

Komori, T., \& Saito, T. (2002, November). The secure DHCP system with user authentication. In Local Computer Networks, 2002. Proceedings. LCN 2002. 27th Annual IEEE Conference on (pp. 123-131). IEEE.

Kozaczuk, W. (1984). Enigma: How the German Machine Cipher was Broken, and How it was Read by the Allies in World War Two (Foreign Intelligence Book Series). Lanham, MD: University Publications of America.

Kuo, E. C., Chang, M. S., \& Kao, D. Y. (2018, February). User-side evil twin attack detection using time-delay statistics of TCP connection termination. In Advanced Communication Technology (ICACT), 2018 20th International Conference on(pp. 211-216). IEEE.

Kurose, J. F. (2005). Computer networking: A top-down approach featuring the internet, 3/E. Pearson Education India.

Lan, P. C., Low, T. P., \& Moon, J. (2018). U.S. Patent No. 9,876,655. Washington, DC: U.S. Patent and Trademark Office.

Li, X., Li, S., Hao, J., Feng, Z., \& An, B. (2017, February). Optimal Personalized Defense Strategy Against ManIn-The-Middle Attack. In $A A A I$ (pp. 593-599).

Li, Y., Eastlake 3rd, D., Dunbar, L., Perlman, R., \& Umair, M. (2018). Transparent Interconnection of Lots of Links (TRILL): ARP and Neighbor Discovery (ND) Optimization (No. RFC 8302).

Lindell, Y. (2018). The Security of Intel SGX for Key Protection and Data Privacy Applications.

MAHESWARI, D., KAUSHIKA, A., \& JENIFER, A. A STUDY ON DATA ENCRYPTION AND DECRYPTION USING HILL CIPHER ALGORITHM.

Mallem, S., \& Yahiaoui, C. (2018, March). A Secure, Green and Optimized Authentication and Key Agreement Protocol for IMS Network. In World Conference on Information Systems and Technologies (pp. 1108-1118). Springer, Cham.

Mallem, S., \& Yahiaoui, C. (2018, March). A Secure, Green and Optimized Authentication and Key Agreement Protocol for IMS Network. In World Conference on Information Systems and Technologies (pp. 1108-1118). Springer, Cham.

'MAN IN THE MIDDLE (MITM) ATTACK' (Incapsula Co.), 2016, Retrieved from: https://www.incapsula.com/web-application-security/man-in-the-middle-mitm.html

'Man-in-the-middle attack' (Wikipedia), 2018, Retrieved from: https://en.wikipedia.org/wiki/Man-in-the-middle attack

"man-in-the-middle-attack" (Rapid Web Ser.), Blog Post, 2017, Retrieved from: https://www.thess1store.com/blog/man-in-the-middle-attack/

'man-in-the-middle-attack-mitm' (Techpedia), 2018, Retrieved from: https://www.techopedia.com/definition/4018/man-in-the-middle-attack-mitm

'man-middle-attack' (CA Tech.), 2018, Retrieved from: https://www.veracode.com/security/man-middle-attack

Meyer, U., \& Wetzel, S. (2004, October). A man-in-the-middle attack on UMTS. In Proceedings of the 3rd ACM workshop on Wireless security (pp. 90-97). ACM

Mitseva, A., Panchenko, A., \& Engel, T. (2018). The State of Affairs in BGP Security: A Survey of Attacks and Defenses. Computer Communications.

Naqash, T., Ubaid, F. B., \& Ishfaq, A. (2012, October). Protecting DNS from cache poisoning attack by using secure proxy. In Emerging Technologies (ICET), 2012 International Conference on (pp. 1-5). IEEE. 
Nath, U., Sharma, G., \& Fletcher, W. (2018). U.S. Patent No. 9,992,192. Washington, DC: U.S. Patent and Trademark Office.

Nayak, N., \& Sharma, R. (2018). Designing security and Surveillance System Using GSM Technology. Journal of Network Communications and Emerging Technologies (JNCET) www. jncet. org, 8(4).

Oh, M., Kim, Y. G., Hong, S., \& Cha, S. (2012). ASA: agent-based secure ARP cache management. IET communications, 6(7), 685-693.

Oh, M., Kim, Y. G., Hong, S., \& Cha, S. (2012). ASA: agent-based secure ARP cache management. IET communications, 6(7), 685-693.

Ornaghi, A., \& Valleri, M. (2003). Man in the middle attacks. In Blackhat Conference

Ou, H. H., Hwang, M. S., \& Jan, J. K. (2010). A cocktail protocol with the authentication and key agreement on the UMTS. Journal of Systems and Software, 83(2), 316-325.

Ouafi, K., Overbeck, R., \& Vaudenay, S. (2008, December). On the security of HB\# against a man-in-the-middle attack. In International Conference on the Theory and Application of Cryptology and Information Security (pp. 108-124). Springer, Berlin, Heidelberg.

Paik, M. (2010, February). Stragglers of the herd get eaten: security concerns for GSM mobile banking applications. In Proceedings of the Eleventh Workshop on Mobile Computing Systems \& Applications (pp. 54-59). ACM.

Pansa, D., \& Chomsiri, T. (2008, November). Architecture and protocols for secure LAN by using a softwarelevel certificate and cancellation of ARP protocol. In Convergence and Hybrid Information Technology, 2008. ICCIT'08. Third International Conference on (Vol. 2, pp. 21-26). IEEE

Parne, B. L., Gupta, S., \& Chaudhari, N. S. (2018). ESAP: Efficient and secure authentication protocol for roaming user in mobile communication networks. Sädhanā, 43(6), 89.

Philip, R. (2007). Securing wireless networks from ARP cache poisoning.

Preneel, B., \& Vercauteren, F. Applied Cryptography and Network Security.

Rahim, R. (2017). Man-in-the-middle-attack prevention using interlock protocol method. ARPN J. Eng. Appl. Sci, 12(22), 6483-6487.

Rupprecht, D., Dabrowski, A., Holz, T., Weippl, E., \& Pöpper, C. (2018). On Security Research towards Future Mobile Network Generations. IEEE Communications Surveys \& Tutorials.

Saif, S., Gupta, R., \& Biswas, S. (2018). Implementation of Cloud-Assisted Secure Data Transmission in WBAN for Healthcare Monitoring. In Advanced Computational and Communication Paradigms (pp. 665-674). Springer, Singapore.

Salim, H., Li, Z., Tu, H., \& Guo, Z. (2012, October). Preventing ARP spoofing attacks through gratuitous decision packet. In Distributed Computing and Applications to Business, Engineering \& Science (DCABES), 2012 11th International Symposium on (pp. 295-300). IEEE

SAMSUNG ELECTRONICS SUSTAINABILITY REPORT 2017 (https://images.samsung.com/is/content/samsung/p5/global/ir/docs/Samsung Electronics Sustainability Report 2017.pdf)

Saxena, N., \& Chaudhari, N. S. (2014). Secure-AKA: An efficient AKA protocol for UMTS networks. Wireless personal communications, 78(2), 1345-1373.

Saxena, N., \& Chaudhari, N. S. (2014, March). NS-AKA: An improved and efficient AKA protocol for 3G (UMTS) networks. In International conference on advances in computer science and electronics engineering (CSEE'14), Kuala Lampur, Malaysia (pp. 220-224).

Schuckers, S. A. (2002). Spoofing and anti-spoofing measures. Information Security technical report, 7(4), 5662.Oh, Myeongjin, Y-G

Scott, L. (2001, March). Anti-spoofing \& authenticated signal architectures for civil navigation systems. In Proceedings of the 16th International Technical Meeting of the Satellite Division of The Institute of Navigation (ION GPS/GNSS 2003)(pp. 1543-1552).

Senie, D., \& Ferguson, P. (1998). Network ingress filtering: Defeating denial of service attacks which employ IP source address spoofing. Network.

Shulman, H. (2018). Implications of Vulnerable Internet Infrastructure. In Digital Marketplaces Unleashed (pp. 921-935). Springer, Berlin, Heidelberg.

Siergiejczyk, M., \& Rosiński, A. (2018, July). Analysis of Information Transmission Security in the Digital Railway Radio Communication System. In International Conference on Dependability and Complex Systems (pp. 420-429). Springer, Cham.

Sinor, D. (2018). U.S. Patent No. 9,965,645. Washington, DC: U.S. Patent and Trademark Office. 
Sounthiraraj, D., Sahs, J., Greenwood, G., Lin, Z., \& Khan, L. (2014). Smv-hunter: Large scale, automated detection of ssl/tls man-in-the-middle vulnerabilities in android apps. In In Proceedings of the 21st Annual Network and Distributed System Security Symposium (NDSS'14.

Stiansen, T. (2018). U.S. Patent No. 9,923,914. Washington, DC: U.S. Patent and Trademark Office.

Stiansen, T., Perlstein, A., \& Foss, S. (2018). U.S. Patent No. 9,942,250. Washington, DC: U.S. Patent and Trademark Office.

Su, X., Wang, Z., Liu, X., Choi, C., \& Choi, D. (2018). Study to Improve Security for IoT Smart Device Controller: Drawbacks and Countermeasures. Security and Communication Networks, 2018.

Su, Z., Timmermans, W., Zeng, Y., Schulz, J., John, V. O., Roebeling, R. A., ... \& Swinnen, E. (2018). An overview of European efforts in generating climate data records. Bulletin of the American Meteorological Society, 99(2), 349-359.

Subashini, S., \& Kavitha, V. (2011). A survey on security issues in service delivery models of cloud computing. Journal of network and computer applications, 34(1), 1-11

Sun, D. Z., Mu, Y., \& Susilo, W. (2018). Man-in-the-middle attacks on Secure Simple Pairing in Bluetooth standard V5. 0 and its countermeasure. Personal and Ubiquitous Computing, 22(1), 55-67.

Trabelsi, Z., \& El-Hajj, W. (2007, June). Preventing ARP attacks using a fuzzy-based stateful ARP cache. In Communications, 2007. ICC'07. IEEE International Conference on (pp. 1355-1360). IEEE.

Truedsson, M., \& Hjelm, V. (2018). Situation-aware Adaptive Cryptography.

Tung, Y. C., Shin, K. G., \& Kim, K. H. (2016, July). Analog man-in-the-middle attack against link-based packet source identification. In Proceedings of the 17th ACM International Symposium on Mobile Ad Hoc Networking and Computing(pp. 331-340). ACM.

Usman, K., Richard, A. T., Moses, A. D., \& Pius, U. T. (2018). A Novel Approach to Enhance the Security of Keys Shared by Users in WLAN Environments Using 3DES Algorithm. International Journal of Advanced Studies in Computers, Science and Engineering, 7(2), 1-7.

Valluri, M. R. (2018). Cryptanalysis of Xinyu et al.'s NTRU-lattice based key exchange protocol. Journal of Information and Optimization Sciences, 39(2), 475-479.

Vidal, C., \& Choo, K. K. R. (2018). Situational Crime Prevention and the Mitigation of Cloud Computing Threats. In Security and Privacy in Communication Networks: SecureComm 2017 International Workshops, ATCS and SePrIoT, Niagara Falls, ON, Canada, October 22-25, 2017, Proceedings 13 (pp. 218-233). Springer International Publishing.

Wallace, Brian Michael, and Jonathan Wesley Miller. "Endpoint-based man in the middle attack detection using multiple types of detection tests." U.S. Patent 9,680,860, issued June 13, 2017.

Wang, X., Zhou, H., Su, J., Wang, B., Xing, Q., \& Li, P. (2018). T-IP: A self-trustworthy and secure Internet protocol. China Communications, 15(2), 1-14.

Weiser, S., Spreitzer, R., \& Bodner, L. (2018, May). Single Trace Attack Against RSA Key Generation in Intel SGX SSL. In Proceedings of the 2018 on Asia Conference on Computer and Communications Security (pp. 575-586). ACM.

'What is a Man In The Middle attack?' (Symantec Corp.), Norton Security Blog, 2018, Retrieved from: https://us.norton.com/internetsecurity-wifi-what-is-a-man-in-the-middle-attack.html

'What is UMTS?' (Tech Target Web), Blog Post, 2018, Retrieved from: https://searchmobilecomputing.techtarget.com/definition/UMTS

Xiang, L., Ng, D. W. K., Schober, R., \& Wong, V. W. (2018). Cache-enabled physical layer security for video streaming in backhaul-limited cellular networks. IEEE Transactions on Wireless Communications, 17(2), 736751.

Xie, M., May, R. A., Yang, J., \& Marwah, K. (2016). U.S. Patent Application No. 14/882,769.

Zhang, D., Zhou, Y., \& Zhang, Y. (2018). A Multi-Level Cache Framework for Remote Resource Access in Transparent Computing. IEEE Network, 32(1), 140-145.

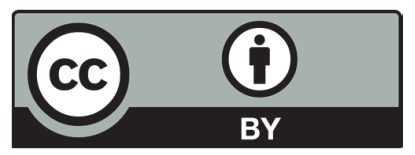

(C) 2019 by the authors; licensee Growing Science, Canada. This is an open access article distributed under the terms and conditions of the Creative Commons Attribution (CCBY) license (http://creativecommons.org/licenses/by/4.0/). 